“ (C) 2013 IEEE. Personal use of this material is permitted. Permission from IEEE must be obtained for all other uses, in any current or future media, including

reprinting/republishing this material for advertising or promotional purposes, creating new collective works, for resale or redistribution to servers or lists, or reuse of any copyrighted component of this work in other works." 


\section{Enhancing Lifetime and Quality of Data in Cluster-based Hierarchical Routing Protocol for Wireless Sensor Network}

\author{
Mian Ahmad Jan, Priyadarsi Nanda, Xiangjian He \\ Centre for Innovation in IT Services and Applications \\ (iNEXT) \\ University of Technology, Sydney \\ Sydney, Australia \\ Mian.A.Jan@student.uts.edu.au \\ \{Priyadarsi.Nanda, Xiangjian.He\}@uts.edu.au
}

\author{
Ren Ping Liu \\ CSIRO ICT Centre \\ Marsfield, Australia \\ ren.liu@csiro.au
}

\begin{abstract}
Wireless Sensor Network (WSN) performs energyextensive tasks and it is essential to rotate sensor nodes frequently so that Cluster Head selections can be made efficiently. In this paper, we aim to improve the lifetime of sensor network by using LEACH based protocols and efficiently utilizing the limited energy available in these sensor nodes. In sensor network, the amount of data delivered at the base station is not important but it is the quality of the data which is of utmost importance. Our proposed approach significantly improves the life time and quality of data being delivered at the base station in sensor network. We evaluate our proposed approach using different sets of node energy levels and in each case our approach shows significant improvement over existing cluster-based hierarchical routing protocols. We evaluate our scheme in terms of energy consumption, life time and quality of data delivered at the base station.
\end{abstract}

Keywords: Wireless Sensor Network, LEACH, Cluster Head, Base Station.

\section{INTRODUCTION}

Recent advances in Micro-Electro-Mechanical Systems (MEMS) technology have enabled the development of miniaturized sensor nodes [1]. These nodes have scarce resources in terms of energy, battery, available bandwidth, memory etc. Wireless Sensor Network (WSN) is a collection of such tiny nodes which gather data from their vicinity of deployment. The data is routed to the Base Station (BS) which then interacts directly with the user. In WSN, it is not feasible to replace the batteries of sensor nodes especially when they are deployed in a hostile environment. Hence, special considerations need to be in place in order to efficiently utilize the energy of the nodes. Energy-efficient routing protocols should be designed carefully to maximize the lifetime of these networks. Latest research in the field of WSN has enabled the development of smart sensor nodes which are very small in size (in order of millimeter cubic) and consume comparatively very low energy in order of tens of Nano joules. In fact, this development is based on the vision of ubiquitous computing [2] that future computing devices will merge so much with their environment that they become almost invisible to the users.
Cluster-based hierarchical routing protocols [3] are the most energy-efficient among all the routing protocols [4]. Low-Energy Adaptive Clustering Hierarchical (LEACH) protocol and its variants effectively use limited energy of the nodes by organizing them into clusters. These protocols operate in rounds (iterations) in which one node in each Cluster is elected as Cluster Head $(\mathrm{CH})$, which performs data aggregation and transmits the aggregated data to the base station. Each node selects a random number between 0 and 1 and if the random number is less than the threshold value then it is elected as a $\mathrm{CH}$ for that specific round. Threshold value varies in each round and depends on the value of the current round. Clustering protocols such as $\mathrm{LEACH}$ reduces energy dissipation by a factor of eight compared with conventional routing protocols like Data-Centric protocols [5].

Cluster-based routing protocols emphasize on improving threshold values for election of $\mathrm{CH}$ nodes. However, improving threshold values does not necessarily elect optimal number of $\mathrm{CHs}$ in each round. Hence, it is highly probable that none of the nodes will be elected as $\mathrm{CHs}$ or all sensor nodes will be elected as $\mathrm{CHs}$ in a specific round due to the distributed and random nature of these protocols. In the former, all the sensor nodes choose random numbers which are greater than the threshold value. In the latter, all the nodes choose random numbers which are less than threshold value. Such situations can result in high energy consumption, low quality of aggregated data and waste of allocated bandwidth and network resources. Each routing protocol aims to improve the lifetime of sensor network in one way or another. This is due to the fact that these nodes run on small batteries with low power.

In this paper, we propose a modified model for $\mathrm{CH}$ selection which improves threshold values and also elects an optimal number of $\mathrm{CHs}$ in each round. The aim of our work is to ensure that the life time of sensor network is extended and also, high quality data is delivered at the base station. Data aggregation function is used which reduces the quantity of data being transmitted to the base station without compromising its quality. 
Our paper is organized into six sections. In Section II, we present related works on Cluster Head selection and refine our objectives for an improved approach. In section III, we present the network architecture of our proposed approach. In Section IV, we present our scheme for Cluster Head selection by improving threshold values. Evaluation and comparison of our scheme with existing approaches are presented in section V. Finally, we conclude our paper in Section $\mathrm{V}$ with future directions.

\section{RELATED WORK}

In this section, a brief overview of various cluster-based routing protocols is presented. There exist many researches on clustering protocols. We present the most notable approaches below.

Low-Energy Adaptive Clustering Hierarchical protocol (LEACH) was presented in [6]. This protocol lays the foundation for clustering protocols. In LEACH protocol, each node chooses a random number between 0 and 1 in each round and if this number is less than the threshold value, it is elected as Cluster Head $(\mathrm{CH})$ node. Threshold value determines the eligibility of a node competing to become $\mathrm{CH}$ in a specific round. Threshold value ranges between 0 and 1 in each round depending on the values of parameters of Equation 1 for LEACH protocol. Once a node is elected as $\mathrm{CH}$, it then advertises itself to other nodes in its vicinity. All the nodes after receiving the advertisement message calculate the signal strength and make a decision whether to join this $\mathrm{CH}$ or not. $\mathrm{CH}$ advertisement is a small message containing a $\mathrm{CH}$ identity and a header which distinguishes it as an announcement message [9]. Non-CH nodes will receive $\mathrm{CH}$ advertisement messages from various $\mathrm{CH}$ nodes. Each non- $\mathrm{CH}$ node then associates itself with a specific $\mathrm{CH}$ node by sending a Join-Request message containing node's ID and its respective $\mathrm{CH}$ node identity. $\mathrm{CH}$ node allocates Time Division Multiple Access (TDMA) slots to each non- $\mathrm{CH}$ node associated with it.

LEACH operates in rounds (iterations) and each round consists of a Setup phase and a Steady-State phase. During Setup phase, Cluster formation and CHs selection take place. Steady-State phase initiates the flow of data to the base station via these $\mathrm{CH}$ nodes. The $\mathrm{CH}$ s aggregate data of the nodes in their respective clusters before transmitting it to $\mathrm{BS}$. The $\mathrm{CH}$ nodes are rotated in each round and hence results in a different set of $\mathrm{CH}$ nodes in subsequent rounds. Selection of $\mathrm{CH}$ is based on the threshold value as determined by equation 1 . Nodes elect themselves as $\mathrm{CHs}$ on the basis of optimal percentage of $\mathrm{CH}$ nodes currently present in the network $(p)$ and number of times it has been elected as $\mathrm{CH}$ in the past $1 / p$ rounds.

$$
T(n)= \begin{cases}\frac{p}{1-p\left(r \bmod \left(\frac{1}{p}\right)\right)} & n \in G \\ 0 & \text { Otherwise }\end{cases}
$$

Here, $p$ is the optimal percentage of $\mathrm{CHs}$ in each round, whose value ranges from five to ten percent of the total number of sensor nodes present in the network and $n$ is the node wishing to become $\mathrm{CH}$ for the current round and $r$ is the current round. $G$ is the set of nodes which have not been elected as $\mathrm{CHs}$ in the past $1 / p$ rounds. Also, $n$ must belong to $G$ otherwise it cannot be elected as $\mathrm{CH}$. Figure 1 represents formation of clusters and their corresponding CHs.

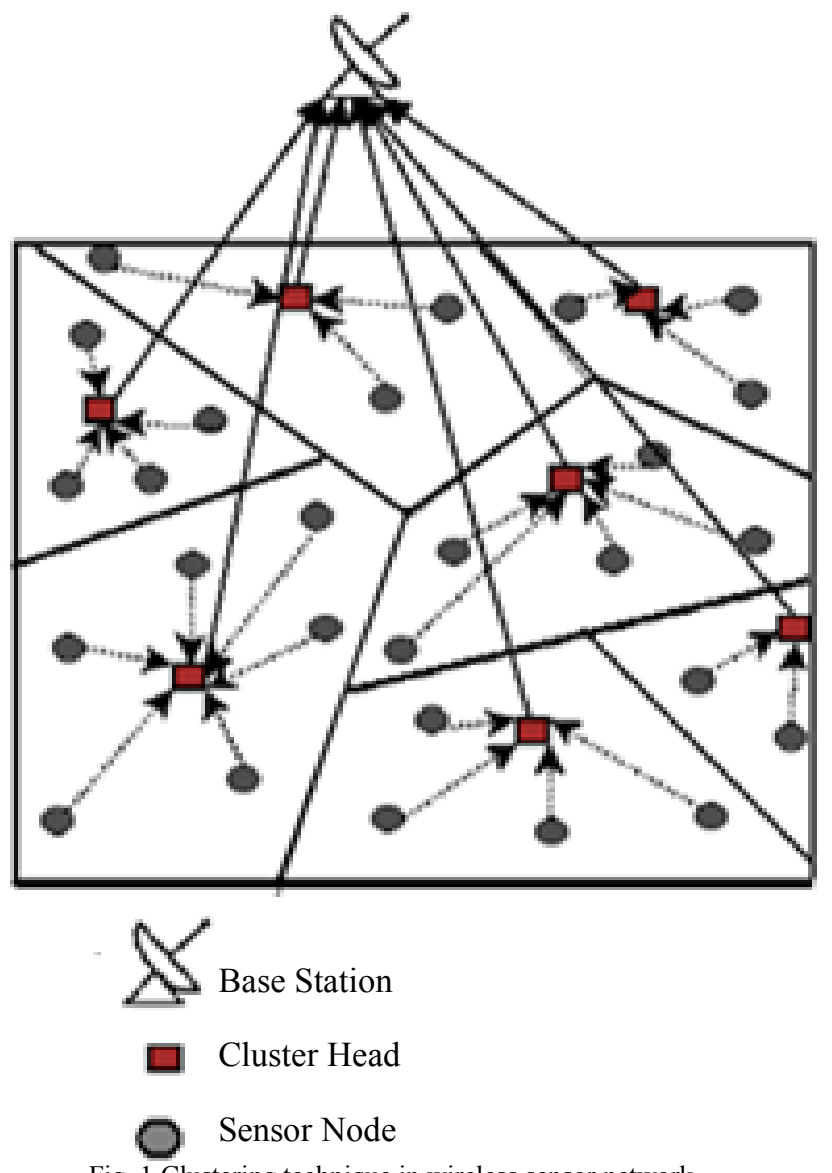

Fig. 1.Clustering technique in wireless sensor network

The problem with $\mathrm{LEACH}$ protocol is that it does not guarantee an optimal number of $\mathrm{CHs}$ in each round. Even nodes with lower energy levels can be elected as $\mathrm{CH}$ in the presence of nodes with higher energy levels. This is due to the fact that Equation 1 which is used to calculate the threshold value in LEACH protocol does not consider the inclusion of node's residual energy. Nodes with lower energy need to refrain themselves from the election process selecting $\mathrm{CHs}$ in order to conserve their energy and increase lifetime of the sensor network. 
The works in $[7,8]$ presented schemes to improve the lifetime of LEACH protocol by including current energy level of the nodes in Equation 1. This inclusion reduces the threshold value $T(n)$, relative to the node's remaining (current) energy level. Hence, the above Equation 1 is modified to LEACH CURRENT and is given below:

$$
\begin{aligned}
& T(n) \text { Current } \\
& =\left\{\begin{array}{l}
\frac{p}{1-p\left(r \bmod \left(\frac{1}{p}\right)\right)} *\left(\frac{E_{\text {current }}}{E_{\text {max }}}\right) \quad n \in G \\
0 \quad \text { Otherwise }
\end{array}\right.
\end{aligned}
$$

Here, $E_{\text {current }}$ is the current energy of the node in a specific round and $E_{\max }$ is its initial energy at the time of network deployment

This equation significantly improves the life time of LEACH protocol because of the inclusion of residual energy of the node in the above threshold equation. However, it works well when the energy levels of the nodes are higher. With the passage of time, the residual energy level of the nodes decreases and hence the threshold value also gets too low. Hence, it becomes a challenging task for the nodes to be elected as $\mathrm{CHs}$ due to lower threshold value.

In Section III, network architecture for our proposed approached is illustrated followed by Cluster Head selection in Section IV, which yields better threshold values with different set of inputs. Our proposed approach not only improves the selection of $\mathrm{CHs}$ but also enhances the life time of sensor nodes with better quality of aggregated data delivered at the base station.

\section{NETWORK ARCHITECTUREOF PROPOSED APPROACH}

The network architecture of our proposed model is based on the following assumptions [6]:

- Base Station is located far away from sensor field and is immobile.

- Sensor nodes are energy-constrained and have the same initial energy.

- Communication channels are symmetric in nature i.e. energy consumed in both the transmission and reception processes are the same. Sensor nodes have the capabilities to adjust their transmission power.

- Sensor nodes sense the environment at a fixed time interval and always have got data to send.

In sensor network, life time of sensors depend on the communication pattern among the nodes of the network. In these networks, most of the energy is consumed in communication rather than processing and sensing [1]. Distances between two adjacent nodes determine the type of communication model to be used. If the distance between two nodes is less than crossover distance $d_{0}$, freespace model $\left(f_{S}\right)$ is used. Otherwise, multipath $(\mathrm{mp})$ model is used. In free space model, there is a line of sight connection between the transmitter and the receiver. In multipath model, signal travel through multiple paths due to deflection through obstacles. Crossover distance is calculated using Equation 3.

$$
d_{0}=\sqrt{\frac{E_{f s}}{E_{m p}}} .
$$

Here $E_{f s}$ is the energy consumed by the amplifier of the transmitter sensor node in free space model and $E_{m p}$ is the energy consumed in multipath model.The radio model used by the sensor nodes in our network is shown in Figure 2 and is based on [6].

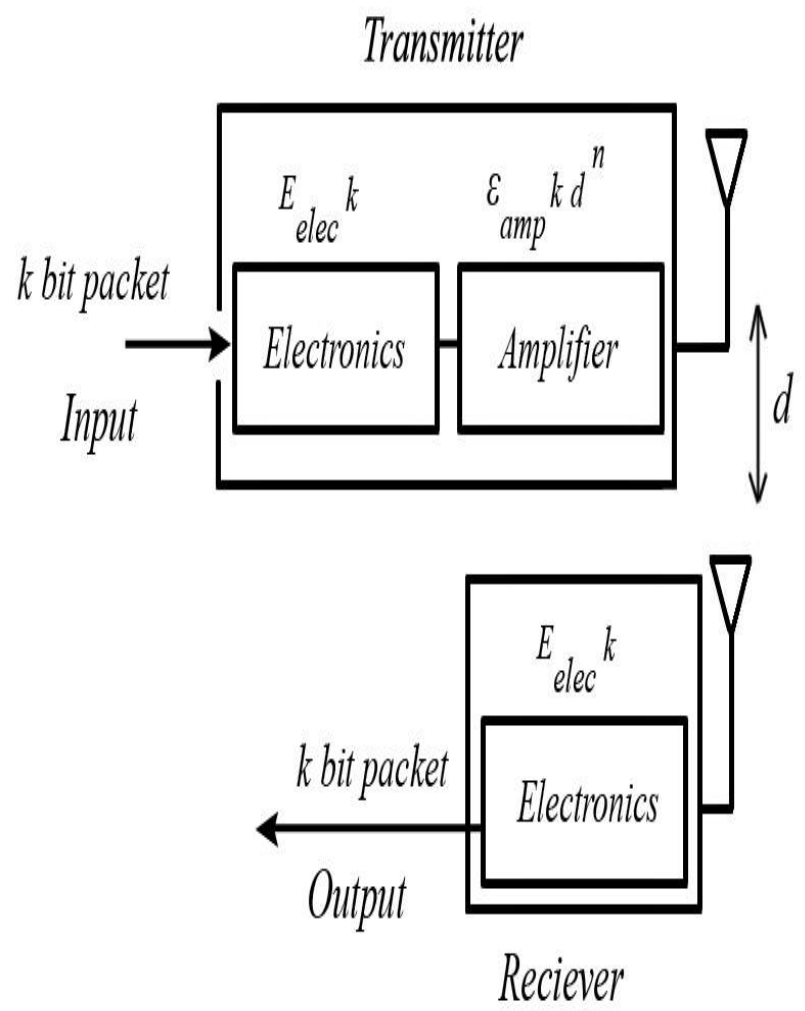

Fig.2. Radio Model for Sensor Node

In Figure 2, $k$ is the length of message and $d$ is the distance between the receiver and transmitter nodes. The value of path loss $(n)$ depends on the distance between two adjacent nodes. $E_{\text {elec }}$ is the energy consumed by the electronic component of the transmitter or receiver node while $E_{m p}$ is the energy consumed by the amplifier component of the transmitting node. Total amount of energy consumed by transmitter node $\left(E_{T X}\right)$ and the receiver node $\left(E_{R X}\right)$ are shown in Equations 4 and 5 respectively. 


$$
\begin{aligned}
E_{T X}(k, d) & \\
& = \begin{cases}k \cdot E_{\text {elec }}+k \cdot E_{f S} d^{2}, & d<d_{0} \\
k \cdot E_{\text {elec }}+k \cdot E_{m p} d^{4} & d \geq d_{0}\end{cases} \\
E_{R X}(k) & =k \cdot E_{\text {elec }}
\end{aligned}
$$

\section{PROPOSED APPROACH FOR IMPROVING LIFE TIME OF LEACH PROTOCOL}

In this Section, we propose an optimized solution for improving lifetime of sensor network by minimizing the consumption of energy across the sensor nodes. Life time of the network is defined as the maximum period of time that nodes are alive after they are deployed in WSN field. We calculate the death of first and the last node over specified rounds. The reason for calculating the death of first and last node is to determine the complete life time of the WSN.

In our proposed approach, we use Equation 6 for $\mathrm{CH}$ selection.

\section{$T(n)_{\text {Proposed }}$}

$$
= \begin{cases}\frac{p}{1-p\left(r \bmod \left(\frac{1}{p}\right)\right)} * E_{\text {con }} & n \in G \\ 0 & \text { Otherwise }\end{cases}
$$

Here, $E_{c o n}$ is the consumed energy of the node and is equal to $E_{\max }-E_{\text {current }}$.

Consumed energy of the nodes is used as a factor for $\mathrm{CH}$ selection in original LEACH equation. Also, in our scheme, each node selects a random number between 0 and 1; and if that number is less than the threshold value of Equation 6, it is elected as $\mathrm{CH}$. The amount of energy consumed by $\mathrm{CH}$ node is based on its distance from BS. If the distance between any node and the BS is less than the crossover distance, then, Equation 7 will be used otherwise equation 8 will be used as shown below.

$$
\begin{aligned}
& E_{\text {con }}=k \cdot E_{\text {elec }}+k \cdot E_{D A}+k \cdot E_{f s} d^{2} \\
& E_{\text {con }}=k \cdot E_{\text {elec }}+k \cdot E_{D A}+k \cdot E_{m p} d^{4}
\end{aligned}
$$

Each Cluster Head node dissipates a certain amount of energy in data processing, data aggregation and transmission to BS. Here, $E_{\text {elec }}$ is the amount of energy dissipated by the electronic component of the $\mathrm{CH}$ node which is used to process the data of its clustering members. $E_{D A}$ is the energy consumed in data aggregation while $E_{m p}$ and $E_{f s}$ are the energy consumed by the amplifier component of the $\mathrm{CH}$ in free space and multipath models respectively. Amplifier of the $\mathrm{CH}$ node transmits the aggregated data to the Base Station using either $E_{m p}$ or $E_{f s}$ based on its distance from BS.
Our proposed approach significantly improves the amount of data transmitted between the CHs and the BS. End users are only interested in a brief description of activities of interest (events) which occur in the vicinity of sensor nodes.Sensor network collects large amount of data which is highly correlated if nodes are close to each other. A large amount of similar data will be redundant at the BS. For this purpose, data aggregationis used at each $\mathrm{CH}$ node [9] by compressing the data in order to reduce the number of packets which are to be delivered at BS. During data aggregation, the quality of data is intact. Once aggregated, the data is delivered to the BS. Based on our proposed scheme, we conduct a set of experiments to validate our propositions and are presented in Section V.

\section{EXPERIMENTAL RESULTS \& ANALYSIS}

We conduct a set of experiments which are based on our proposed approach and compare our approach with existing LEACH and its variants. The experiments were conducted using the following parameters.

- Number of nodes in the network $=100$

- Maximum energy of each node $E_{\max }=0.5 \mathrm{~J}$

- Optimal Probability of $\mathrm{CH}$ selection $=0.1$

- $\quad$ Number of Rounds $=5000$

- Data Aggregation Energy $\left(E_{D A}\right)=5 \mathrm{~nJ}$

- Energy Consumed in transmission and reception per message $\left(E_{\text {elec }}\right)=50 \mathrm{~nJ}$

- Length of message (Packet) $k=4000$ bits

The main aim of our proposed scheme is to extend the life time of the network by minimizing the consumption of energy by the sensor nodes without compromising the quality of data being delivered to the base station. Life time of a network is determined by the total number of rounds that the network remains active and functional.

We calculate the number of dead nodes in each round, first dead node, last dead node, amount of data delivered to $\mathrm{CHs}$ and BS and the quality of data received at the BS.We compare our proposed approach with LEACH and LEACH CURRENT using the above mentioned parameters. First Node Dead (FND) indicates the start of decline in network's life time and Last Node Dead (LND) indicates the end of network's life time. The network starts to decline when its first node dies. The FND metric indicates the duration for which the sensor network is fully functional, i.e. none of the sensor node failure occurs due to battery outage [10].

Figure 3 below presents information on the First Node Dead (FND). In this figure, the first node dies in round 874 in our proposed approach as compared to LEACH and LEACH CURRENT which stands for 850 and 775 rounds respectively. 


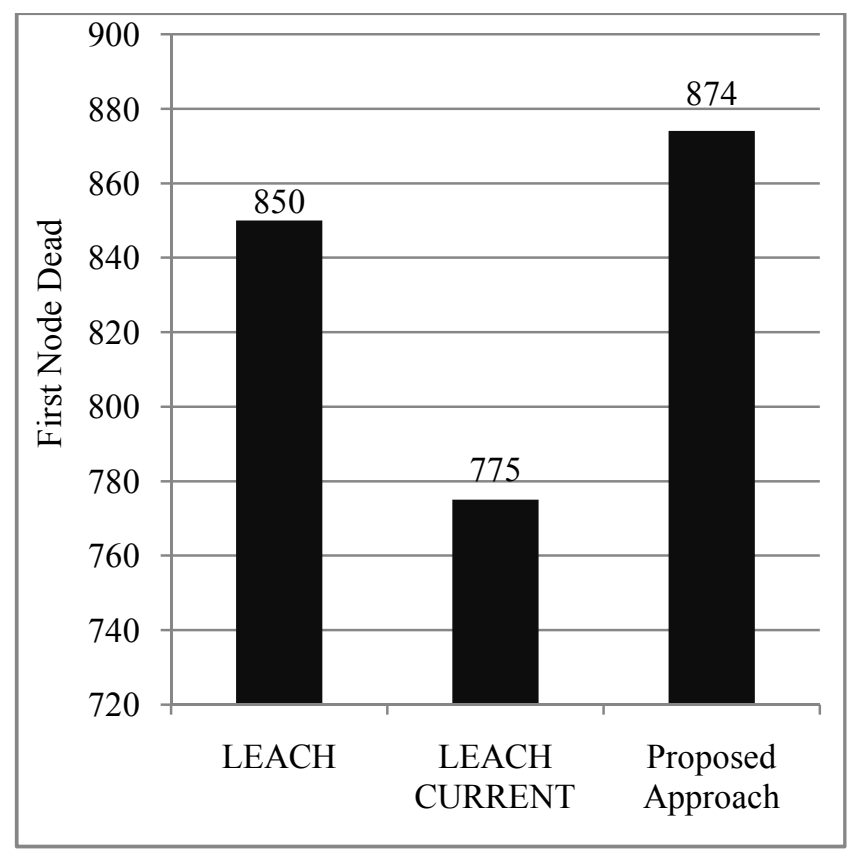

Fig. 3.First Node Dead vs. Number of Rounds

Our proposed approach slightly improving over the existing schemes is due to the fact that in our scheme, all the nodes start with the same amount of energy at the time of deployment due to the homogenous nature of LEACH and LEACH CURRENT protocols.

Once last node dies, the network becomes nonoperational and the Last Node Dead (LND) is shown in Figure 4.

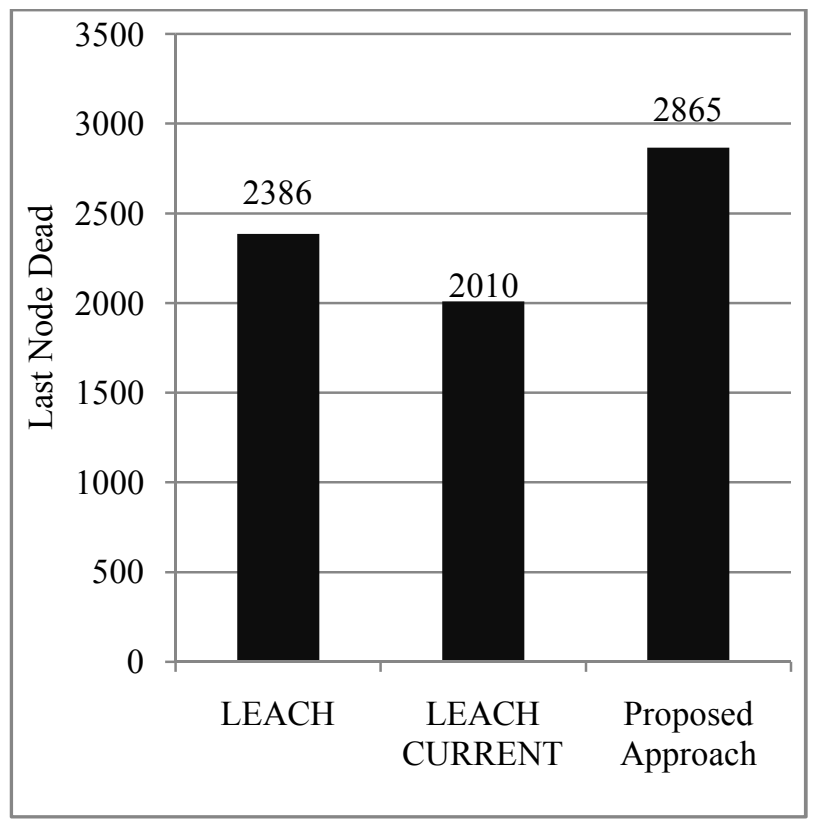

Fig.4. Last Node Dead vs. Number of Rounds

In terms of LND, our proposed approach shows significant improvement over existing schemes. In our scheme, the network dies in round number 2865 , which is around $20 \%$ to $40 \%$ improvement over LEACH and LEACH CURRENT respectively. These results significantly improve the life time of our proposed cluster protocol assuming that nodes in the above scenario have only half joule of energy.

The number of packets delivered at $\mathrm{CH}$ nodes in each round is shown in Figure 5.

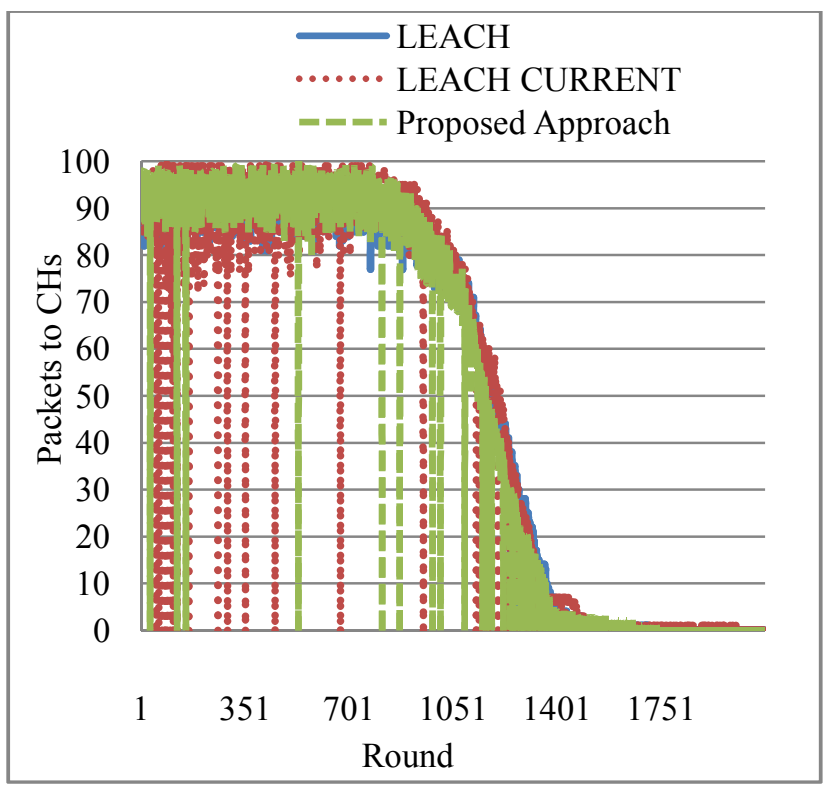

Fig.5. Number of Packets Delivered at Cluster Heads in

Each Round

In LEACH protocol, large amount of data packets are transmitted to the $\mathrm{CH}$ nodes which roughly receive 80 to 95 packets in most rounds as shown in the above figure. In LEACH CURRENT, there is a high drop in packet transmission to the $\mathrm{CHs}$ from round number 50 to round 150 . On the other hand, our proposed approach constantly transmits data to the $\mathrm{CH}$ nodes during all these rounds, and in most of the time there are more than 80 packets transmitted to $\mathrm{CHs}$ in each round.Hence, the total amount of data transmitted to $\mathrm{CHs}$ in our scheme is higher compared with existing approaches. We have considered up to 2000 rounds so that the graph can be visible better and can clearly differentiate among the three approaches. Total amount of data collected at $\mathrm{CHs}$ over the span of network life time is shown in Figure 7 which is directly derived from Figure 5.

The number of packets delivered at the Base Station (BS) is shown in figure 6. To make the graph better visible and understand clearly, we have considered up to 2500 rounds below. The reason is that after this number of rounds, all the three approaches transmit packets infrequently as the nodes are very low on energy at that point of time. Even in this figure after round number 1500 , one or two data packets are transmitted abruptly as shown below. 


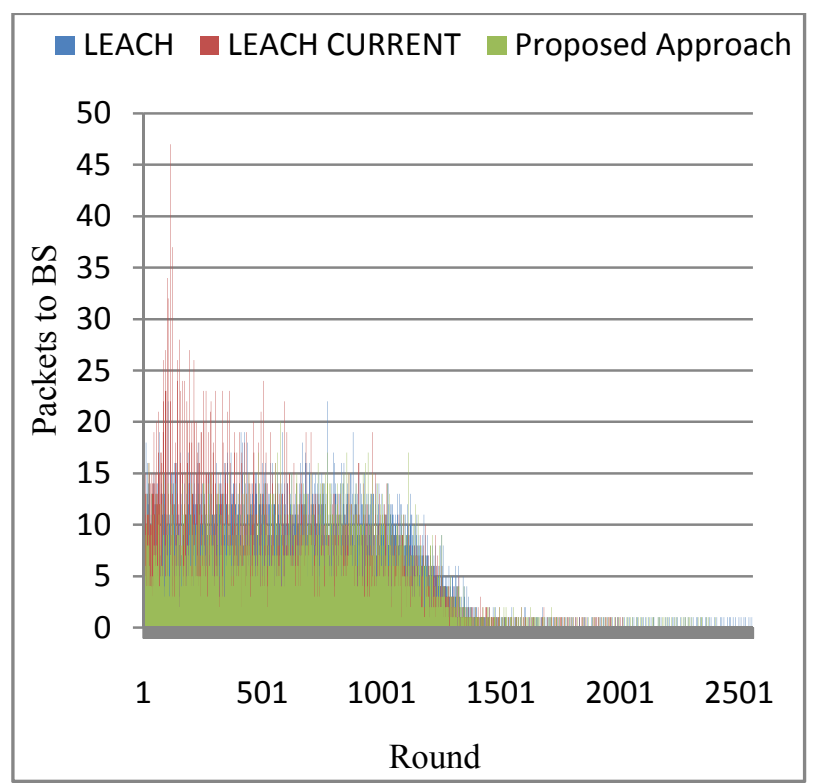

Fig.6. Number of Packets Delivered at Base Station

In this figure, LEACH CURRENT abruptly delivers a large number of packets in the start which results in depletion of energy of the sensor nodes in this protocol and hence the network dies early.

We have considered the number of data packets transmitted to $\mathrm{CHs}$ and BS in the Figures 5 and 6 up to a certain number of rounds (2000 rounds for Figure 5 and 2500 rounds for Figure 6 respectively) for the sake of clarity and visibility of the graphs. After these numbers of rounds, transmission of data packetsis very low on order of few packets ( 1 or 2 in each round), which are transmitted intermittently as well.

The total amount of data packets aggregated by the $\mathrm{CH}$ nodes over the span of network life time is shown in Figure 7.

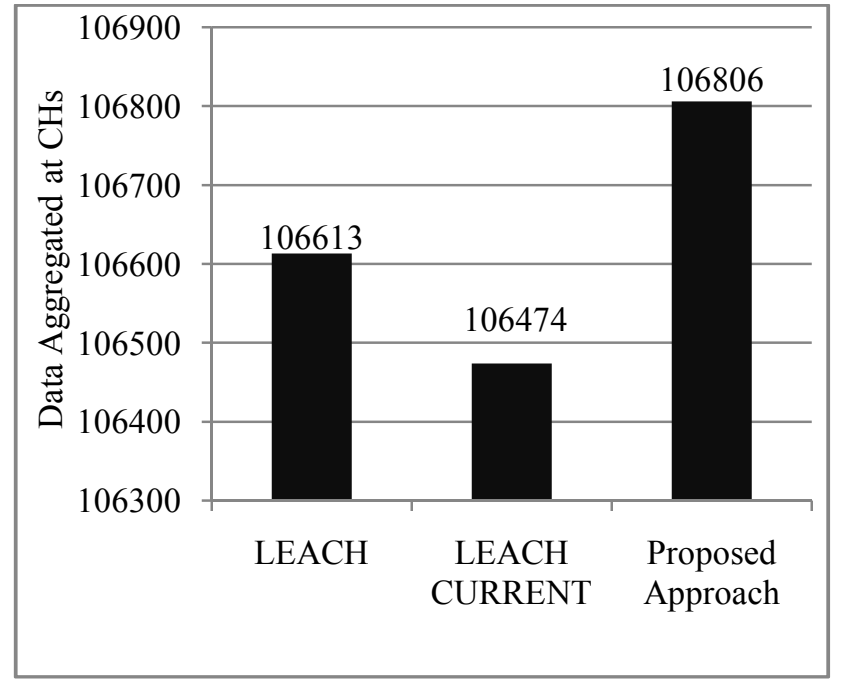

Fig.7. Data Aggregated at the Cluster Head Nodes
The amount of data aggregated at the base station is shown in Figure 8.

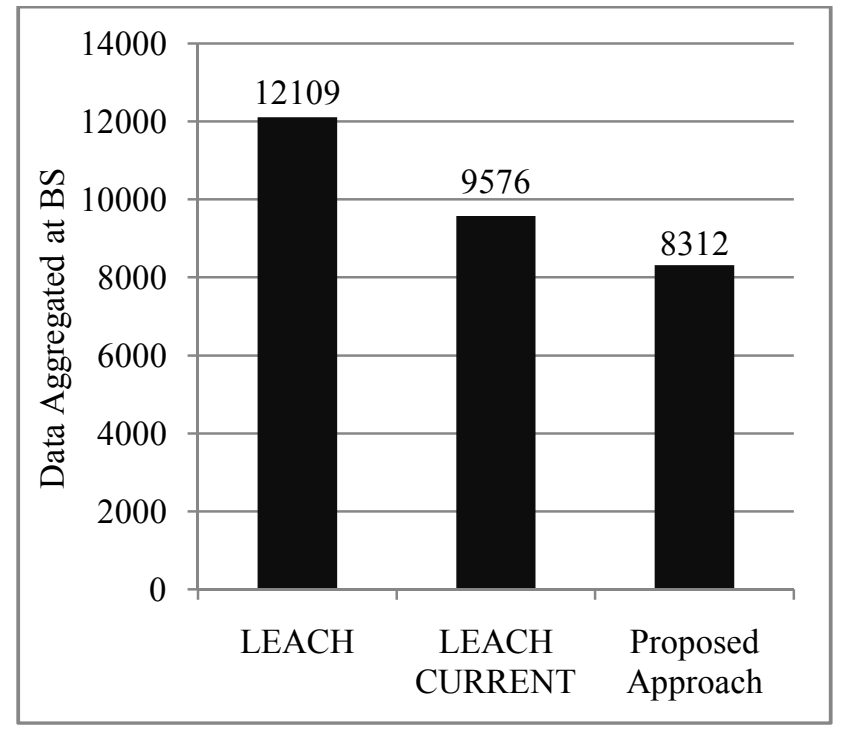

Fig.8: Data Aggregated at the Base Station

Finally, the quality of data is shown in Figure 9 which is measured using Equation 9 below:

\section{Quality of Data \\ $=($ Sum of Data to BS/Sum of data to CHs $) * 100$}

Quality of data is the amount of data transmitted by $\mathrm{CHs}$ to the base station after data aggregation. In order to obtain high quality data at the BS, fewer number of data packets need to be delivered at the BS as mentioned above. Quality of data is directly related to delay, energy efficiency, congestion control, reliability and other quality of service metrics. As WSN are constraint by energy resources, it is utmost important that data should be aggregated properly by the $\mathrm{CHs}$ so that only few packets are then transmitted to the BS. Fewer Packets (or messages) will not only reduce energy consumption but will also make sure that other Quality of Service (QoS) metrics are achieved. Lower number of packets means low delay, high reliability and low congestion probability in the network.

Quality of data is a percentage value and is shown below in Figure 9 which is derived based on Figures 7 and 8 respectively. In this figure, quality of data for our proposed approach is 7.78, which means that in each round; for every 100 packets (received at the Cluster Heads), only 7.78 on an average are transmitted to the base station. LEACH CURRENT transmits on average 8.99 packets out of 100 packets to the Base Station while LEACH delivers 11.36 packets out of 100 to the Base Station. The lower the percentage is, the higher quality of data delivered to BS for end user is. 


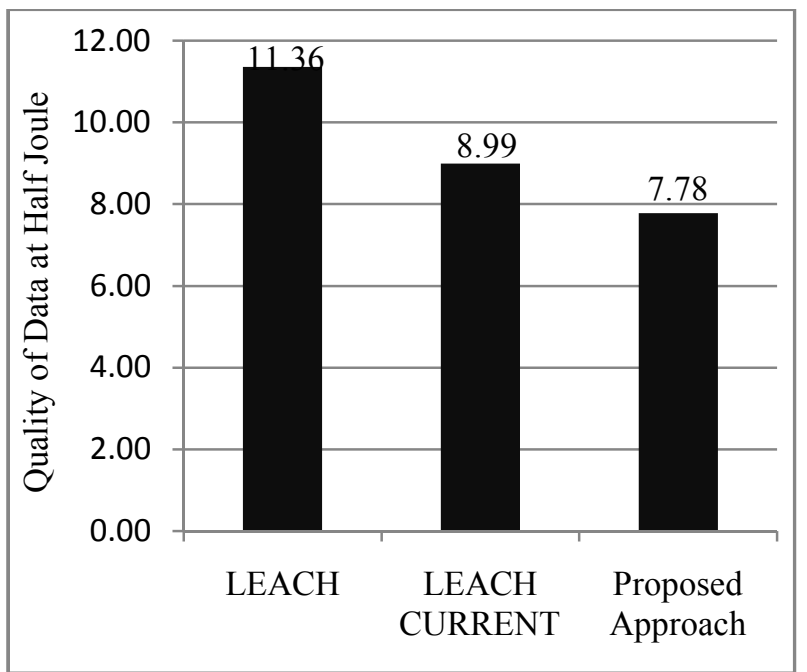

Fig.9. Quality of Data at Half Joule of Energy

We also perform our simulation with another set of values and increase energy level for the sensor nodes to $1.0 \mathrm{~J}$ and the number of rounds to 10000 . We obtain the following results with much higher improvement over existing protocols. In fact as the number of rounds and the amount of energy of the nodes increase, the performance of our approach improves too. In Figure 10, quality of data is shown using node energy level as 1 Joule and number of rounds as 10000 keeping the optimal selection of $\mathrm{CH}$ selection as $0.1(p)$.

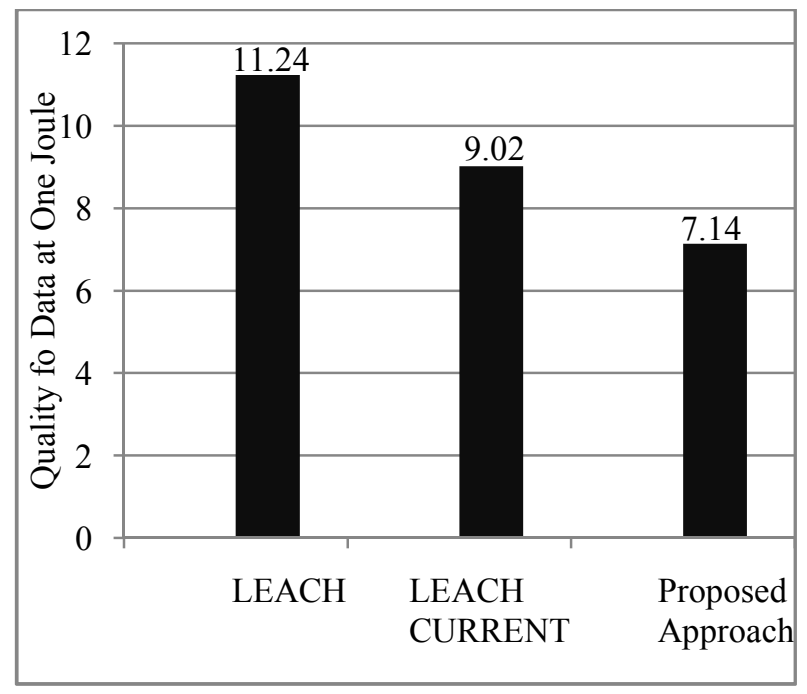

Fig.10. Quality of Data at One Joule Energy

This figure shows that our proposed approach delivers only 7.14 packets on average to $\mathrm{BS}$ in each round. LEACH CURRENT transmits 9.02 packets on average in each round and LEACH protocol transmits 11.24 packets. Changing energy of nodes to 1 Joule, we have achieved significant improvement in quality of data compared to LEACH CURRENT and LEACH. Our network has only 100 nodes but in real sensor network there are thousands of nodes. These improvements in terms of quality of data will save a large amount of energy as a lot fewer packets will be transmitted.

\section{CONCLUSION}

In this paper, we have proposed a scheme for improving the life time of LEACH protocol (ultimately Sensor network's lifetime). Life time has been measured in terms of first and last node death in the network. The Amount of data delivered at the $\mathrm{BS}$ and $\mathrm{CHs}$ have been calculated and the quality of data delivered at the BS has been presented. In sensor network, redundancy is a major issue since nodes are located close to each other and it is highly probable that nodes located in the vicinity of each other will gather similar data. This issue not only consumes a considerable amount of energy but also leads to compromise on other QoS metrics such as delay, congestion, packet loss, blocking probability etc. Our aim is to efficiently aggregate the data at the $\mathrm{CH}$ nodes so that only a small amount of data packets are transmitted to BS. This will not only improve the QoS metrics but also enhance life time of the nodes in terms of energy efficiency. In this paper, we have presented simulation results for homogenous nodes because LEACH and LEACH CURRENT are assumed to be homogenous in nature. As we are directly comparing our proposed scheme against the LEACH and LEACH CURRENT, which consider equal energy levels across all sensor nodes at the start, we have used the same assumption in our proposed work. However, we would like to emphasize that the energy levels across the nodes are variant with time and it is obvious that all sensor nodes cannot retain the same energy levels throughout their life time. That is why, referring to Figure 4, the node energy recedes over the time and the level of their engagement in the network. Hence, at the end, the nodes will have different energy levels. Our proposed scheme has a strong performance over the existing schemes and is different in many aspects. In future, we aim to evaluate our approach for mobile CHs nodes, mobile sensor nodes and mobile sink to derive a performance metric for quality of service, amount of data transmitted at $\mathrm{BS}$ and $\mathrm{CHs}$, packet drop probability, latency etc.

\section{REFRENCES}

[1]. J.Yick, B. Mukherjee and D.Ghosal, "Wireless sensor network survey," Computer Networks., vol. 52, no. 12,pp. 2292-2330, 2008.

[2]. G. Bell andP. Dourish,“'Yesterday's tomorrows; notes on ubiquitous computing's dominant vision,"Personal and Ubiquitous Computing, vol. 11, no. 2,pp. 133143,2006.

[3]. O. Boyinbode, L. Hanh, A. Mbogho, M. Takizawa andR. Poliah, "A Survey on Clustering Algorithms for Wireless Sensor Networks," 13th International Conference onNetwork-Based Information Systems (NBiS), pp. 358-364, 2010. 
[4]. S. Nikolidakis, D. Kandris, D. Vergados, andC. Douligeris, "Energy Efficient Routing in Wireless Sensor Networks Through Balanced Clustering", Algorithms, vol. 6, no. 1,pp. 29-42, 2013.

[5]. K. Akkaya and M. Younis, "A survey on routing protocols for wireless sensor networks," Ad Hoc Networks, vol. 3, no. 3,pp. 325-349, 2005.

[6]. W.R. Heinzelman, A. Chandrakasan and H. Balakrishna, "Energy-efficient communication protocol for wireless microsensor networks," Proceedings of the 33rd Annual Hawaii International Conference on System Sciences, vol.2, pp.1-10, 2000.

[7]. M.J, Handy, M. Haase andD.Timmermann,"Low energy adaptive clustering hierarchy with deterministic cluster-head selection,"Fourth International Workshop on Mobile and Wireless Communications Network., pp. 368-372, 2002.

[8]. Y.Fan and J. Yu, "'The Communication Protocol for Wireless Sensor Network about LEACH,'CISW 2007 International Conference on

ComputationalIntelligence and Security Workshops,

pp. 550-553, 2007

[9]. W.R.Heinzelman, "Application-Specific Protocol Architectures for Wireless Networks" PhD. Dissertation, Massachusetts Institute of Technology, 2000.

[10]. Y. Chen, E. Chan and S. Han, "Energy Efficient Multipath Routing in Large Scale Sensor Networks with Multiple Sink Nodes," Springer, Vol. 3756, pp. 390-399, 2005 